\title{
Conocimientos sobre VIH/SIDA en adolescentes urbanos: consenso cultural de dudas e incertidumbres
}

Ramiro Caballero-H oyos, Dr en CS, ${ }^{(1)}$ Alberto Villaseñor-Sierra, MD, D r Sc, ${ }^{(2)}$

\section{Caballero-Hoyos R, Villaseñor-SierraA. Conocimientos sobreVIH/SIDA en adolescentes urbanos: consenso cultural de dudas e incertidumbres. Salud Publica Mex 2003;45 supl 1:S108-S114.}

\begin{abstract}
Resumen
Objetivo. Describir conocimientos sobreVIH/SIDA en adolescentes de distintos estratos so cio económicos, mediante un análisis de consenso cultural. Material y métodos Estudio transversal muestral descriptivo y correlacional. Cuestionario aplicado a 758 adolescentes de Guadalajara,Jalisco, México, entre julio de 1995 y marzo de 1996. Análisis: a) consenso por estratos y género con análisis factorial, b) estimación de patrón cultural de respuestas correctas y de concordancia de respuestas individuo/grupo por correlaciones $r$ de Pearson. Resultados. Hubo altos grados de consenso en todos los estratos. La concordancia de conocimientos individuo/grupo fue mayor en estratos superiores. El patrón de respuestas correctas mostró: a) conocimientos similares sobre formas de transmisión casual, características del SIDA y alto riesgo en trabajadoras del sexo comercial, y b) diferencias basadas en dudas e incertidumbres so bre la protección del condón, la distinción VIH/SIDA y la transmisión en clínicas. Conclusiones Los conocimientos son homogéneos en todos los estratos y se orientan hacia un modelo biomédico. Sin embargo, contienen elementos de duda e incertidumbre que precisan de intervenciones informativas segmentadas.
\end{abstract}

Palabras clave:VIH/SIDA; conocimientos; consenso cultural; adolescencia; clases sociales; México

\author{
Caballero-Hoyos R, Villaseñor-Sierra A. \\ Knowledge of HIV/AIDS among urban adolescents: \\ cultural consensus of doubts and uncertainties \\ Salud Publica Mex 2003;45 suppl 1:S108-S114.
}

\begin{abstract}
A bstract
Objective.To describe the knowledge of HIV/AID S among adolescents from different socioeconomic strata (SS), using cultural consensus analysis. Material and Methods. A cross-sectional descriptive and analytic study was conducted from July 1995 to March 1996, among 758 adolescents from Guadalajara,M éxico. D ata were collected using a questionnaire, and analyzed by means of:a) consensus by SS and gender using factorial analysis; and b) identification of the cultural pattern of correct answers, and concordance of individual/group answers using Pearson's $r$ correlation coefficients. Results. There was a high degree of consensus across SS. The individual/group knowledge concordance was greater in higher SS. The pattern of correct responses showed:a) similar knowledge on casual transmission, AIDS characteristics, and higher risk in sex workers; and b) discrepancies regarding doubts and uncertainties about condom protection, distinction between HIV and AID S, and HIV transmission in health clinics. Conclusions. Knowledge was homogeneous and mainly medically oriented across all SS. $\mathrm{N}$ evertheless, there were elements of doubt and uncertainty that require differentiated informative interventions.
\end{abstract}

Key words: HIV/AID S; knowledges; cultural consensus; adolescense; so cial class; Mexico

Este trabajo es parte del proyecto número 4136P-S9607 financiado por el Consejo N acional de Ciencia y Tecnología de México (CO N ACyT).

(1) Unidad de Investigación Epidemiológica y en Servicios de Salud del Adolescente. Instituto Mexicano del Seguro Social. Guadalajara, Jalisco, México.

(2) Centro de Investigación Biomédica de 0 ccidente. Instituto Mexicano del Seguro Social. G uadalajara, Jalisco, México.

Fecha de recibido: 18 de abril de 2001 • Fecha de aprobado: 11 de abril de 2002

Solicitud de sobretiros: Dr. Ramiro Caballero-Hoyos. Unidad de Investigación Epidemiológica y en Servicios de Salud del Adolescente. A venida Tonalá 121, Tonalá 45400, Unidad Médica Familiar N 0.93, IMSS, Tonalá, Jalisco, México.

Correo electrónico: rchsur@ terra.com.mx. 
E 1 desarrollo de la epidemia del VIH/SIDA está asociado con desigualdades sociales expresadas en accesos diferenciales a oportunidades de protección y cuidado de la salud, en diferentes contextos socioeconómicos. La Organización Mundial de la Salud (OMS) estimó para el año 2000 unos 40 millones de infectados con el VIH en el mundo, de los cuales más de $90 \%$ vivían en países pobres, donde las tasas de infección tenderán a aumentar por la deficiente oferta de sistemas sanitarios y los limitados recursos preventivos y asistenciales. ${ }^{1}$

La epidemia del VIH/SIDA también se asocia con desigualdades de clase social debido a que la prevalencia del VIH y la incidencia del SIDA son mayores en áreas donde la población pertenece a estratos socioeconómicos inferiores, como es el caso de México. ${ }^{2}$

Las diferencias de género son otra expresión de las desigualdades asociadas con la epidemia, puesto que son un componente central en las posibilidades de lograr posiciones socioeconómicas de mayor o menor privilegio. Estas diferencias implican una mayor probabilidad de que los hombres transmitan el VIH a sus parejas sexuales mujeres ${ }^{3} \mathrm{y}$ en mayor medida en contextos de pobreza.

La epidemia ha tenido efectos importantes en la población adolescente y joven de América Latina y El Caribe. La Organización Panamericana para la Salud (OPS) estimó en 1997 que la mitad de todas las nuevas infecciones se presentaban en personas menores de 25 años de edad y que la mayoría se infectaba por la vía sexual. $^{4}$

En México se reportaron 32802 casos de SIDA de marzo de 1983 al 1 de octubre de 1997, con una razón hombre/mujer de 6:1. Para ese mismo periodo, Jalisco, estado cuya capital es la ciudad de Guadalajara, era la tercera entidad federativa con más casos $(11.5 \%$ del total) y con una tasa de incidencia de 65.4 por 100000 habitantes. Del total de casos del país, 1.6\% se ubicaron en el grupo de adolescentes de 15 a 19 años de edad, grupo en el cual predominaba la vía de transmisión sexual. ${ }^{5}$

El porcentaje de casos en adolescentes de 15 a 19 años de edad reconocidos con SIDA en ese periodo debe asociarse con el porcentaje de casos informados en el grupo de 20 a 29 años de edad que abarcaba $30 \%{ }^{5} \mathrm{La}$ asociación debe apreciar la existencia de un periodo prolongado de latencia entre la seroconversión en VIH y las manifestaciones del SIDA que puede durar entre dos y 11 años, ${ }^{6}$ lo cual llevaría a pensar que una importante proporción de casos del grupo de 20 a 29 años de edad pudo haberse infectado con el VIH durante su adolescencia.
Algunos estudios que aportan datos sobre conocimientos del VIH/SIDA en adolescentes y jóvenes urbanos de México, tuvieron hallazgos diferenciales. Uno de estos estudios reportó niveles altos de conocimientos en población abierta, ${ }^{7}$ estudiantes ${ }^{8,9}$ homosexuales y bisexuales varones, ${ }^{10}$ mientras que otros hallaron niveles regulares de conocimientos en población abierta ${ }^{11}$ y estudiantes. ${ }^{12}$ Los distintos hallazgos mencionaron que existía un menor grado de conocimientos sobre formas de transmisión del VIH.

La calificación del nivel de conocimientos en esos estudios se hizo sobre la base del porcentaje de respuestas correctas en escalas prefijadas por investigadores, en función de su correspondencia con modelos biomédicos considerados como válidos. Al contrario de esos estudios, en el presente trabajo se aplicará como criterio de evaluación de conocimientos un modelo de consenso cultural, ${ }_{1}^{13}$ orientado a identificar si hay o no un sistema homogéneo de conocimientos sobre el VIH/ SIDA, a partir de las respuestas originales por parte de los informantes a un cuestionario estructurado.

El modelo de consenso cultural es una aproximación formal que define el consenso como un patrón alto de correspondencia en las respuestas de los informantes, de un mismo grupo cultural, a las preguntas de un cuestionario referido a un tema específico. En este sentido, es un modelo que estima: a) la verosimilitud de que un sistema homogéneo de conocimientos predomine en una cultura; b) el grado de correspondencia entre los conocimientos de cada individuo y el sistema cultural de conocimientos, y c) el patrón de respuestas aceptadas como correctas o válidas en el grupo cultural, sin referencia a un patrón biomédico de respuestas correctas. ${ }^{13}$

En tal sentido los propósitos del presente estudio, aplicado a adolescentes de cuatro estratos socioeconómicos de Guadalajara, fueron: a) estimar el grado de consenso cultural de conocimientos sobre el VIH/ SIDA, en cada estrato y por géneros; $b$ ) describir el nivel de competencias culturales en cada estrato por género, y c) estimar el patrón de respuestas correctas de cada uno de los estratos y establecer el grado de correlación entre esos patrones.

\section{Material y métodos}

Se realizó un estudio transversal por encuesta muestral aplicada a 758 adolescentes de 15 a 19 años de edad, en cuatro estratos socioeconómicos de Guadalajara, entre julio de 1995 y marzo de 1996. Los estratos socioeconómicos (alto, medio, bajo y marginado) fueron definidos según la residencia de los adolescen- 
tes en Areas Geoestadísticas Básicas (AGEBs) de la Zona Metropolitana de Guadalajara, tipificadas según diferencias observables en indicadores físicos de vivienda, acceso a servicios y ambiente barrial. ${ }^{14}$

El modelo de consenso cultural propone que en el estudio de patrones culturales, el tamaño de muestra no debe ser grande, debido a que la correlación promedio entre informantes tiende a ser alta (0.5 o más). Sugiere un tamaño mínimo de 30 informantes en cada estrato para clasificar 99\% de las preguntas correctamente, bajo un promedio de competencia cultural esperada (concordancia entre las respuestas individuales y el patrón estimado de respuestas correctas del grupo) de 0.5 y un nivel de confianza de $0.95 .{ }^{15}$ Sin embargo, se elevó la muestra en cada estrato para encontrar -por muestreo aleatorio- variación en las características de género, edad, nivel de instrucción y ocupación, en los entrevistados.

Las entrevistas se hicieron en los hogares de las y los adolescentes. Se obtuvo por escrito el consentimiento informado de los padres o tutores y la participación de los entrevistados se sujetó a su aceptación voluntaria. El porcentaje de rechazo a la entrevista fue menor a $5 \%$.

El cuestionario estuvo compuesto por 24 preguntas de conocimientos sobre el VIH/SIDA adoptadas de un cuestionario sobre conocimientos, actitudes y prácticas del Programa Global de SIDA de la OMS. ${ }^{16}$ Las preguntas abordaron conceptos, formas de transmisión, consecuencias y formas de prevención. Cada pregunta tuvo cinco categorías de respuesta ordinales ("definitivamente cierto" a "definitivamente falso").

El análisis de la información se efectuó en dos momentos: 1) se generó un modelo de consenso cultural con un procedimiento ajustado a respuestas ordinales, ${ }_{17}^{17}$ para cada estrato socioeconómico y por géneros, mediante un análisis factorial de componentes principales que agrupa a los individuos en función de sus respuestas. El criterio de bondad de ajuste para la obtención del consenso en el modelo fue que el primer factor tuviera una razón tres veces mayor a la del segundo factor, lo cual implica una alta concordancia en las respuestas de los informantes. ${ }^{18}$ 2) A su vez, el modelo estimó, por un lado, un patrón de respuestas correctas a las preguntas en cada estrato socioeconómico, en función de las intercorrelaciones de las respuestas originales. Este patrón se expresó en porcentajes correspondientes a las categorías de respuestas estimadas como correctas en cada estrato socioeconómico y pruebas de significancia ji cuadrada. A efectos de obtener la correlación entre patrones, se calcularon coeficientes rho Spearman y sus pruebas de significancia. Por otro lado, también el modelo estimó la competencia cultural individual por géneros (definida como el coeficiente promedio de concordancia entre las respuestas individuales y el patrón estimado de respuestas correctas del grupo), dentro de cada estrato socioeconómico. En forma complementaria, se calculó el nivel de concordancia en las respuestas de los informantes en cada grupo, elevando al cuadrado el valor promedio de la competencia cultural. Los datos considerados en el análisis fueron los códigos de respuesta originales, sin una calificación previa de respuestas correctas e incorrectas. El análisis se procesó con los paquetes ANTHROPAC v. 4.1. y SPSS v.9.

\section{Resultados}

De 758 entrevistados, $50 \%$ fueron hombres y $50 \%$ mujeres. Según edades, 20.5\% fueron de 15 años, 20.1\% de $16,19.9 \%$ de $17,20.3 \%$ de 18 y $19.2 \%$ de 19 (edad promedio $=17.1 \pm 1.5)$. Según estratos socioeconómicos, $20.8 \%$ fueron del estrato alto, $26.4 \%$ del medio, $26.4 \%$ del bajo y $26.4 \%$ del marginado. Según ocupación, $38.6 \%$ estudiaban, $28.9 \%$ trabajaban, $20.1 \%$ trabajaban y estudiaban y $12.4 \%$ no trabajaban ni estudiaban (diferencias por estratos: $p<0.001)$. Según nivel de instrucción, $47.7 \%$ estaban en preparatoria, $39.9 \%$ en secundaria y escuelas técnicas, $11.2 \%$ en primaria, $11.2 \%$ en universidad y $1.2 \%$ no tenían instrucción (diferencias por estratos: $p<0.001$ ).

El análisis de consenso cultural mostró conocimientos relativamente homogéneos en todos los estratos $\mathrm{y}$, dentro de cada estrato, en ambos géneros. El criterio teórico de bondad de ajuste del modelo de consenso se cumplió en todos los casos: las razones entre el factor $1 \mathrm{y}$ el factor 2 fueron mayores a tres. Conviene señalar, sin embargo, que los niveles de consenso fueron más altos en los estratos superiores: estrato alto $=7.8$, estrato medio $=5.9$, estrato bajo $=0.45$ y estrato marginado $=3.5$ (cuadro I).

A su vez, los coeficientes promedio de competencia cultural fueron mayores en los estratos alto (0.78) y medio (0.75) y menores en los estratos bajo (0.70) y marginado (0.67), lo cual indica la presencia de una mayor homogeneidad a medida que el estrato era más alto. Los coeficientes fueron casi similares en ambos géneros, dentro de todos los estratos.

Finalmente, los promedios de concordancia grupal también fueron mayores en los estratos más altos: estrato alto $=0.61$, estrato medio $=0.56$, vs. estrato bajo $=0.49$, y estrato marginado $=0.45$.

El cuadro II describe el patrón cultural de conocimientos correctos estimado por el modelo de consenso cultural para cada estrato. El mismo permite apreciar patrones muy similares en todos los estratos, 


\section{Cuadro I \\ Consenso de CONOCIMIENTOS SOBRE EL VIH/SIDA: RAZÓN Y VARIANZA ACUMULADA DE FACTORES, PROMEDIO DE COMPETENCIAS CULTURALES Y CONCORDANCIA GRUPAL SEGÚN ESTRATOS SOCIOECONÓMICOS. Guadalajara, Jalisco, México 1995-1996}

\begin{tabular}{|c|c|c|c|c|c|c|c|}
\hline $\begin{array}{l}\text { Estratos socioeconómicos } \\
\text { y géneros }\end{array}$ & $\begin{array}{l}\text { Razón del } \\
\text { factor } 1\end{array}$ & $\begin{array}{l}\% \text { varianza } \\
\text { acumulada }\end{array}$ & $\begin{array}{l}\text { Razón del } \\
\text { factor } 2\end{array}$ & $\begin{array}{l}\text { \% varianza } \\
\text { acumulada }\end{array}$ & Razón F1:F2 & $\begin{array}{l}\text { Competencia cultural } \\
\text { (M edia } \pm \text { DS) }\end{array}$ & $\begin{array}{c}\text { Concordancia } \\
\text { grupal* }\end{array}$ \\
\hline Alto $(n=158)$ & 11 & $86.50 \%$ & 1.4 & $94.50 \%$ & 7.8 & $0.78+0.14$ & 0.61 \\
\hline Hombres ( $n=79)$ & 11.1 & $87.00 \%$ & 1.5 & $94.80 \%$ & 7.4 & $0.79 \pm 0.12$ & 0.62 \\
\hline Mujeres ( $n=79)$ & 10.9 & $86.30 \%$ & 1.4 & $94.20 \%$ & 7.8 & $0.78 \pm 0.16$ & 0.61 \\
\hline Medio $(n=200)$ & 9.5 & $85.30 \%$ & 1.6 & $94.30 \%$ & 5.9 & $0.75 \pm 15$ & 0.56 \\
\hline Hombres ( $n=100)$ & 9.8 & $86.10 \%$ & 1.7 & $94.80 \%$ & 5.8 & $0.76 \pm 0.15$ & 0.58 \\
\hline Mujeres $(n=100)$ & 9.1 & $84.60 \%$ & 1.5 & $93.90 \%$ & 6.1 & $0.75 \pm 0.16$ & 0.56 \\
\hline Bajo $(n=200)$ & 7.2 & $81.60 \%$ & 1.6 & $93.00 \%$ & 4.5 & $0.70 \pm 0.16$ & 0.49 \\
\hline Hombres ( $n=100)$ & 7.1 & $82.10 \%$ & 1.8 & $93.70 \%$ & 3.8 & $0.71 \pm 0.16$ & 0.5 \\
\hline Mujeres $(n=100)$ & 7.3 & $81.10 \%$ & 1.4 & $92.30 \%$ & 5.2 & $0.70 \pm 0.17$ & 0.49 \\
\hline Marginado $(n=200)$ & 5.6 & 79.2 & 1.6 & $91.70 \%$ & 3.5 & $0.67 \pm 0.21$ & 0.45 \\
\hline Hombres $(n=100)$ & 5.9 & $81.10 \%$ & 1.6 & $91.90 \%$ & 3.7 & $0.67 \pm 0.21$ & 0.45 \\
\hline Mujeres ( $n=100)$ & 5.4 & $77.40 \%$ & 1.7 & $91.60 \%$ & 3.2 & $0.67 \pm 0.22$ & 0.45 \\
\hline
\end{tabular}

puesto que hubo concordancia de respuestas en 17 de 24 preguntas (cuadro II). Estos patrones tuvieron una importante similaridad con las respuestas correctas del modelo biomédico: rho Spearman promedio $=0.75$, $p<0.001$ (estrato alto $=0.81$, estrato medio $=0.88$, estrato bajo $=0.67$ y estrato marginado $=0.65$ ).

La similaridad en los patrones de respuesta estimados fue mayor entre los estratos socioeconómicos más parecidos: estrato alto con estrato medio (rho Spearman=0.97, $p<0.000)$, y estrato bajo con estrato marginado ( $r$ ho Spearman=0.93, $p<0.000$ ). Las correlaciones más bajas se dieron al comparar los patrones de los estratos alto y marginado (rho Spearman= $0.63, \mathrm{p}<0.001)$ y medio y marginado. (rho Spear$\operatorname{man}=0.65, p<0.001)$.

Los patrones tuvieron respuestas similares en todos los estratos, en tres aspectos generales: a) formas de transmisión casual del VIH: para todos los estratos, es "definitivamente falso" que hay riesgo de transmisión al trabajar cerca de una persona infectada, al jugar con un niño infectado, al compartir sanitarios con personas infectadas, al compartir vajilla y cubiertos con alguien infectado, al tomar las manos, tocar o besar a alguien infectado y al ser picado por mosquitos que picaron previamente a alguien infectado. También es "definitivamente falso" para todos que el cloro mate al VIH; b) la definición del SIDA como enfermedad mortal: para todos los estratos, es "definitivamente cierto" que el SIDA causa la muerte, disminuye las defensas del organismo y que no hay vacunas disponibles para combatirlo. También es "definitivamente cierto" que el VIH se transmite por la vía sexual, por compartir agujas y jeringas con alguien infectado y por la vía perinatal de una madre infectada a su hijo. En esta definición, predomina en todos la duda sobre si los y las adolescentes podrían tener o no el VIH, y c) el alto riesgo de las trabajadoras del sexo comercial: es "definitivamente cierto" que hay riesgo de transmisión al tener relaciones sexuales no protegidas con las trabajadoras del sexo comercial (cuadro II).

Pese al predominio de las similaridades en las respuestas, hubo diferencias específicas entre los estratos, en cinco aspectos generales: a) la eficacia de la protección del condón: en los estratos alto y medio, "no están seguros" de si el condón previene eficazmente contra el VIH, mientras que en los estratos bajo y marginado piensan que es "probablemente cierto" que prevenga. Un elemento que fomenta esa duda es que en todos los estratos piensan que es "probablemente cierto" que el VIH pasa el hule de los condones. Un detalle sobre el condón en que no concuerdan es que en los estratos bajo y marginado "no están seguros" de si los condones se dañan y caducan, mientras 


\section{Cuadro II \\ Patrón estimado de respuestas correctas sobre VIH/SIDA en adolescentes, por estratos socioeconómicos. Guadalajara, Jalisco, México, 1995-1996 en porcentajes}

\begin{tabular}{|c|c|c|c|c|c|c|}
\hline \multirow[b]{2}{*}{ Preguntas } & \multicolumn{4}{|c|}{ Estratos socioeconómicos y opciones de respuesta correctas } & \multirow[b]{2}{*}{$\chi^{2}(\text { g.l. . })^{\ddagger}$} & \multirow[b]{2}{*}{ Valor $p$} \\
\hline & Alto $(n=158)$ & Medio $(n=200)$ & Bajo $(n=200)$ & Marginado $(n=200)$ & & \\
\hline \multirow{2}{*}{ Existe diferencia entre VIH y SIDA } & D efinitivamente cier to & D efinitivamente cierto & Probablemente cierto & Probablemente cierto & & \\
\hline & 50.6 & 36.1 & 33.4 & 38.9 & $30.2(9)$ & 0.001 \\
\hline \multirow{2}{*}{ VIH causa SIDA } & D efinitivamente cierto & D efinitivamente cierto & Probablemente cierto & Probablemente cierto & & \\
\hline & 87.9 & 81.6 & 76.7 & 73.1 & $29.5(9)$ & 0.004 \\
\hline \multirow[t]{2}{*}{ Persona con VIH se ve saludable } & D efinitivamente cierto & D efinitivamente cierto & Definitivamente falso & Definitivamente falso & & \\
\hline & 43 & 27.4 & 22.3 & 28 & $40.1(9)$ & 0.001 \\
\hline \multirow[t]{2}{*}{ Con mirada se reconoce infectado } & D efinitivamente falso & Definitivamente falso & Probablemente falso & D efinitivamente cierto & & \\
\hline & 58.2 & 60.2 & 61.2 & 55.2 & $29.7(9)$ & \\
\hline
\end{tabular}

SIDA causa muerte

Definitivamente cierto Definitivamente cierto Definitivamente cierto Definitivamente cierto

\begin{tabular}{|c|c|c|c|c|c|c|}
\hline & 93.7 & 91.9 & 87.8 & 89.8 & $10.9(9)$ & 34 \\
\hline$\overline{S I D A}$ disminuye defensas organismo & $\begin{array}{c}\text { D efinitivamente cierto } \\
91.1\end{array}$ & $\begin{array}{c}\text { D efinitivamente cierto } \\
80.7\end{array}$ & $\begin{array}{c}\text { D efinitivamente cierto } \\
71.5\end{array}$ & $\begin{array}{c}\text { D efinitivamente cierto } \\
68.4\end{array}$ & $46.7(9)$ & \\
\hline Hay vacuna disponible contra SIDA & $\begin{array}{c}\text { D efinitivamente falso } \\
86.1\end{array}$ & $\begin{array}{c}\text { D efinitivamente falso } \\
73.5\end{array}$ & $\begin{array}{c}\text { Definitivamente falso } \\
69.6\end{array}$ & $\begin{array}{c}\text { Definitivamente falso } \\
62.6\end{array}$ & $33.4(9)$ & \\
\hline Los adolescentes no tienen SIDA & $\begin{array}{l}\text { Probablemente falso } \\
82.9\end{array}$ & $\begin{array}{l}\text { Probablemente falso } \\
57.3\end{array}$ & $\begin{array}{l}\text { Probablemente falso } \\
47.5\end{array}$ & $\begin{array}{c}\text { Probablemente falso } \\
43.2\end{array}$ & $81.7(9)$ & \\
\hline Infectado transmite VIH por coito & $\begin{array}{c}\text { D efinitivamente cierto } \\
94.9\end{array}$ & $\begin{array}{c}\text { D efinitivamente cierto } \\
88.9\end{array}$ & $\begin{array}{c}\text { D efinitivamente cierto } \\
84.1\end{array}$ & $\begin{array}{c}\text { D efinitivamente cierto } \\
82.1\end{array}$ & 23.1(9) & \\
\hline VIH por compartir agujas y jeringas & $\begin{array}{c}\text { D efinitivamente cierto } \\
98.1\end{array}$ & $\begin{array}{c}\text { D efinitivamente cierto } \\
91.7\end{array}$ & $\begin{array}{c}\text { D efinitivamente cierto } \\
84.7\end{array}$ & $\begin{array}{c}\text { D efinitivamente cierto } \\
83.3\end{array}$ & $36.7(9)$ & \\
\hline Embarazada transmite VIH a hijo & $\begin{array}{c}\text { Definitivamente cierto } \\
82.9\end{array}$ & $\begin{array}{c}\text { D efinitivamente cierto } \\
75.3\end{array}$ & $\begin{array}{c}\text { D efinitivamente cier to } \\
76.9\end{array}$ & $\begin{array}{c}\text { D efinitivamente cierto } \\
69.3\end{array}$ & $22.6(12)$ & \\
\hline Condón previene VIH & $\begin{array}{c}\text { No estoy seguro } \\
36.1\end{array}$ & $\begin{array}{c}\text { No estoy seguro } \\
33.7\end{array}$ & $\begin{array}{c}\text { Probablemente cierto } \\
33.4\end{array}$ & $\begin{array}{c}\text { Probablemente cierto } \\
40.8\end{array}$ & $52.2(9)$ & \\
\hline$\overline{\mathrm{VIH}}$ pasa hule condones & $\begin{array}{c}\text { Probablemente cierto } \\
37.9\end{array}$ & $\begin{array}{c}\text { Probablemente cierto } \\
47.1\end{array}$ & $\begin{array}{c}\text { Probablemente cierto } \\
43.7\end{array}$ & $\begin{array}{c}\text { Probablemente cierto } \\
48.2\end{array}$ & 19.4(9) & \\
\hline Condones se dañan y caducan & $\begin{array}{c}\text { D efinitivamente cierto } \\
65.8\end{array}$ & $\begin{array}{c}\text { D efinitivamente cierto } \\
58.7\end{array}$ & $\begin{array}{c}\text { No estoy seguro } \\
44\end{array}$ & $\begin{array}{c}\text { No estoy seguro } \\
55.2\end{array}$ & $58.0(6)$ & \\
\hline Sexo seguro $=$ no semen $/$ secreciones $*$ & $\begin{array}{c}\text { D efinitivamente falso } \\
29.8\end{array}$ & $\begin{array}{c}\text { Probablemente cierto } \\
36.5\end{array}$ & $\begin{array}{l}\text { Probablemente cierto } \\
42.3\end{array}$ & $\begin{array}{c}\text { Probablemente cierto } \\
46.7\end{array}$ & $29.7(9)$ & \\
\hline
\end{tabular}

Riesgo sexo prostituta sin condón Definitivamente cierto Definitivamente cierto Definitivamente cierto Definitivamente cierto

\begin{tabular}{|c|c|c|c|c|c|c|}
\hline & 94.3 & 90,0 & 86.8 & 87.6 & 28.1(12) & 0.021 \\
\hline \multirow[t]{2}{*}{ Riesgo clínica dental o centro de salud } & Probablemente cierto & Probablemente cierto & D efinitivamente cierto & D efinitivamente cierto & & \\
\hline & 31 & 23.3 & 25.9 & 26.1 & $22.2(12)$ & 0.102 \\
\hline \multirow[t]{2}{*}{ Riesgo trabajar cerca de infectado } & D efinitivamente falso & Definitivamente falso & D efinitivamente falso & Definitivamente falso & & \\
\hline & 80.4 & 76.5 & 76.5 & 75.6 & 26.9(12) & 0. \\
\hline \multirow[t]{2}{*}{ Riesgo jugar con niño infectado VIH } & Definitivamente falso & Definitivamente falso & Definitivamente falso & Definitivamente falso & & \\
\hline & 71.5 & 68.1 & 71.1 & 66.5 & $26.3(12)$ & \\
\hline \multirow[t]{2}{*}{ Riesgo compartir sanitarios infectados } & D efinitivamente falso & D efinitivamente falso & Definitivamente falso & D efinitivamente falso & & \\
\hline & 44.3 & 43.6 & 42.3 & 36.2 & $21.8(12)$ & \\
\hline \multirow[t]{2}{*}{ Riesgo compartir vajilla y cubiertos } & Definitivamente falso & D efinitivamente falso & D efinitivamente falso & Definitivamente falso & & \\
\hline & 56.9 & 61.6 & 58.3 & 56.1 & 29.1(12) & \\
\hline \multirow[t]{2}{*}{ Riesgo tomar manos, tocar o besar } & Definitivamente falso & Definitivamente falso & Definitivamente falso & D efinitivamente falso & & \\
\hline & 78.5 & 77.2 & 72,0 & 70.4 & $32.1(12)$ & \\
\hline \multirow[t]{2}{*}{ Riesgo ser picado por mosquitos } & D efinitivamente falso & D efinitivamente falso & D efinitivamente falso & D efinitivamente falso & & \\
\hline & 52.5 & 41.2 & 38.8 & 34.6 & $26.2(12)$ & \\
\hline
\end{tabular}

El cloro mata el VIH

Definitivamente falso Definitivamente falso D efinitivamente falso D efinitivamente falso $\begin{array}{llllll}60.1 & 58.4 & 57.4 & 51.4 & 16.8(9) & 0.053\end{array}$

* La pregunta completa decía: "El sexo seguro se basa en no dejar que el semen o las secreciones vaginales entren al organismo"

$\neq \chi^{2}(\mathrm{~g} . \mathrm{l})=.\mathrm{ji}$ cuadrada (grados de libertad)

$n=758$ 
que en los estratos alto y medio piensan que sí; b) la distinción entre el VIH y el SIDA: en los estratos alto y medio piensan que es "definitivamente cierto" que hay diferencia entre VIH y SIDA y que el VIH causa el SIDA, mientras que en los estratos bajo y marginado piensan que es "probablemente cierto"; c) la apariencia física del infectado con el VIH: en los estratos bajo y marginado creen que es "definitivamente falso" que las personas con el VIH aparenten estar saludables y que por ello basta con la mirada para reconocer que están infectados. En los estratos alto y medio piensan lo contrario; d) la transmisión en algunos servicios de salud: en los estratos bajo y marginado piensan que es "definitivamente cierto" que hay riesgo de transmisión en clínicas dentales y espacios de salud donde atienden a personas infectadas, mientras que en los estratos medio y alto piensan que es "probablemente cierto", y e) el concepto de "sexo seguro": en el estrato alto se piensa que "es definitivamente falso" que el concepto "sexo seguro" implique solamente no dejar penetrar fluidos sexuales en el organismo, mientras que en los otros estratos piensan que es "probablemente cierto".

\section{Discusión}

En el presente estudio se encontraron altos grados de consenso en los conocimientos sobre el VIH/SIDA, en todos los estratos socioeconómicos y en ambos géneros. Sin embargo, el nivel de consenso fue mayor en los estratos superiores, en los cuales se encontró una mayor homogeneidad de conocimientos basada en coeficientes de competencia cultural más altos.

Los patrones estimados de respuestas correctas tuvieron niveles altos de similaridad entre estratos, pero se encontraron correlaciones más altas entre los patrones de estratos más parecidos en características socioeconómicas. Los patrones de los estratos superiores tuvieron mayor similaridad con el patrón del modelo biomédico.

Los hallazgos de la investigación se pueden confrontar con los reportados por Trotter y colaboradores $^{19}$, quienes aplicaron un modelo de consenso en un estudio intercultural sobre conocimientos del VIH/SIDA en México, Guatemala y la población latina de dos ciudades de Estados Unidos de América. Estos autores encontraron altos grados de consenso en todos los contextos, con niveles más altos de competencia cultural en las ciudades norteamericanas que en México y Guatemala. También hallaron una mayor similaridad del patrón de respuestas correctas en los contextos geográficos más cercanos. Estos hallazgos y los nuestros permiten plantear la posibilidad de en- contrar niveles más altos de consenso y homogeneidad de conocimientos sobre el VIH/SIDA en contextos con mayores recursos socioeconómicos.

En cuanto al patrón de respuestas correctas que estimaron en el mencionado estudio, los aspectos de similaridad interculturales que se asemejan a los que encontramos fueron: a) las posibles formas de transmisión casual no implican riesgo; b) el SIDA como enfermedad mortal incurable causada por el VIH que se transmite por vías sanguínea, sexual y perinatal, y c) el alto riesgo de transmisión en las trabajadoras del sexo comercial y los homosexuales. El estudio, sin embargo, fue más amplio que el nuestro, debido a que consideró también síntomas y tratamientos en la estimación de patrones de respuesta.

Las estrategias de información sobre el VIH/SIDA deberían incidir diferencialmente en los distintos estratos socioeconómicos. El presente análisis de consenso cultural permite plantear algunos aspectos de conocimiento, que precisan ser modificados debido a que pueden incidir en actitudes que propician prácticas de riesgo y estigmatización social: a) las incertidumbres sobre la seguridad que proporciona el uso del condón en los y las adolescentes de todos los estratos; b) la concepción determinista sobre las trabajadoras del sexo comercial como grupo de alto riesgo de transmisión, en todos los estratos; c) la incertidumbre sobre la transmisión del VIH en espacios clínicos donde atienden a personas con el VIH/SIDA, en todos los estratos; d) la distinción conceptual entre el VIH y el SIDA, sobre todo en los estratos bajo y marginado. La poca claridad sobre esta distinción hace que se piense, en los estratos bajo y marginado, que las personas infectadas tienen siempre apariencia de enfermas; y e) la definición conceptual de "sexo seguro", sobre la cual hay dudas en todos los estratos y especialmente en el estrato alto.

Los enfoques tradicionales de evaluación de conocimientos en el VIH/SIDA, basados en premisas conductuales y mediciones estructuradas, han llevado a concluir que en los adolescentes el nivel alto de conocimientos no siempre tiene correspondencia con prácticas saludables ni con la modificación de prácticas de riesgo. ${ }^{20,21}$ Desde el enfoque del modelo del consenso cultural, Dressler y colaboradore ${ }^{22}$ han generado una propuesta de análisis llamada Consonancia Cultural, que pretende establecer el grado de correspondencia entre los modelos de consenso cultural y las prácticas sociales. Encontraron, por ejemplo, en Brasilia, asociaciones independientes entre algunas prácticas de salud (estrés percibido, síntomas de depresión y relacionados con la presión arterial) y los modelos de consenso sobre el apoyo social recibido, en habi- 
tantes de tres estratos socioeconómicos (con coeficientes de correlación más altos que los obtenidos por escalas de apoyo social). Esta forma de análisis podría ser desarrollada como una estrategia complementaria al trabajo ya realizado en este estudio y como una alternativa a los estudios de cambio de comportamiento.

El nivel de análisis del trabajo permitió detectar la homogeneidad de los conocimientos del VIH/ SIDA y estimar patrones culturales de respuestas correctas y su correlación, en los distintos estratos socioeconómicos. A la vez, los patrones de respuesta basados en las escalas ordinales originales, orientaron a encontrar elementos de duda e incertidumbre en los conocimientos similares y diferenciales entre estratos. Sin embargo, la principal limitación del estudio fue mostrar la orientación de los conocimientos, sin profundizar sobre ellos ni hacer la indagación de las condiciones estructurales, los motivos y las creencias que fundamentan el consenso cultural y los patrones culturales estimados. Una profundización de este tipo es necesaria y requiere el diseño de investigaciones cualitativas con componentes etnográficos.

\section{Referencias}

1. Mertens TE, Low-Beer D. HIV and AID S:W here is the epidemic going? Bull W orld Health 0 rgan 1996;74:121-129.

2. González-Block MA, LiguoriA L. El SIDA en los estratos socio-económicos de México. En: Gonzalez-Block MA, Liguori, AL, ed. Perspectivas de Salud Pública. Cuernavaca, Morelos, México. Instituto N acional de Salud Pública, 1992: 21-36.

3. Sherr L.Tomorrow's era: Gender, psychology and HIV infection. En:Sherr L. Hankins C, Bennett L, ed.AIDS as a gender issue. Psychosocial perspectives. London:Taylor Francis, 1996:16-45.

4. 0 rganización Panamericana de la Salud. Salud de los adolescentes. Plan de Acción 1998-2001 sobre la salud y desarrollo del adolescente en las A méricas.W ashington, D.C .: O rganización Mundial de la Salud, 1997.

5. Consejo $\mathrm{N}$ acional para la Prevención y control del SIDA. Epidemiología. Separata de la revista SIDA-ETS. SIDA-ETS 1997;3:1-10.
6. Bowler S, Sheon AR, D 'Angelo LJ, Vermund SH. HIV and AIDS among adolescents in the United States: Increasing risk in the 1990s. J Adolescence 1992;15: 345-371.

7. Population Reference Bureau. La actividad sexual y la maternidad entre las adolescentes de América Latina y El Caribe. Riesgos y consecuencias. Atlanta (GA): Population Reference Bureau, 1992.

8. Izazola J,Valdespino J, Juárez L, M ondragón M, Sepúlveda J. C onocimientos, actitudes y prácticas relacionadas con el SIDA. Bases para el diseño de programas educativos. En: Sepúlveda J, Bronfman M, Ruiz-Palacios G, Stanislawki E, Valdespino JL, coord. SIDA, ciencia y sociedad en México. México, D.F. IN SP-FCE, 1991:297-336.

9. Consejo N acional de Población. Encuesta N acional sobre Sexualidad y Familia en Jóvenes de Educación Media Superior. México, D.F.: Conapo, 1988.

10. Sepúlveda J, coord. Encuesta sobre sexualidad y SIDA en hombres homosexuales y bisexuales. México, D.F.: Secretaría de Salud, 1989.

11. Caballero R,Villaseñor $A$, Hidalgo A. Fuentes de información y su relación con el grado de conocimientos sobre el SIDA en adolescentes de México. Rev Saude Publica 1997;31:351-359.

12. Alfaro L, Rivera S, Díaz-Loving, R. Actitudes y conocimientos hacia la sexualidad y SIDA en estudiantes de preparatoria. Psic Soc Mex 1992;4:95100.

13. Romney AK, W eller SC, Batchelder, W H. Culture as consensus: A theory of culture and informant accuracy. Am Anthropologist 1986; 88: 313-338.

14. Instituto N acional de Estadística, Geografía e Informática. Estratificación socio-económica de la zona Metropolitana de Guadalajara: IN EGI, 1992.

15. W eller SC, Romney AK. Systematic data collection. N ewbury Park: Sage, 1988:69-78.

16. World Health 0 rganization. Social and behavioural research unit. Research package: Knowledge, attitudes, beliefs and practices on AIDS survey. Appendix: Q uestionnaire. W ashington, D.C.:W HO , 1990.

17. Romney AK, Batchelder W H, W eller SC. Recent applications of C onsensus Theory. Am Behav Sci 1987;31:163-177.

18. Weller SC, Mann NC. Assessing rater performance without a gold estandar using ConsensusTheory. Med D ecision Making 1997;17:71-79. 19.Trotter II RT,W eller SC, Baer RD, Pachter LM, G lazer M, García de Alba JE et al. Consensus theory model of AIDS/SIDA beliefs in four Latin populations. AIDS Educ Prev 1999;11: 414-426.

20. Stiffman AR, Earls F, D ore P, C unningham R. C hanges in Acquired Immunodeficiency Syndrome related risk behavior after adolescence: Relationships to knowledge and experience concerning Human Immunodeficiency Virus Infection. Pediatrics 1992;89:950-956.

21. Peruga A, C elentano DD. Correlates of A ID S knowledge in samples of the general population. Soc Sci Med 1993;36:509-524.

22. D ressler W W, Balieiro MC, D os Santos JE. Culture, socioeconomic status and physical and mental health in Brazil. Med Anthropol Res 1996:52:331-353. 